\title{
Meta-Analysis on Safety of Dapagliflozin in Patients with Type 2 Diabetes Mellitus
}

\author{
Lili She, Hongyan Wu \\ Yangtze University Medical School, Department of Endocrinology, Jingzhou First People’s Hospital, Yangtze University, \\ Jingzhou, China \\ Email: 736947197@qq.com
}

How to cite this paper: She, L.L. and $\mathrm{Wu}$, H.Y. (2018) Meta-Analysis on Safety of Dapagliflozin in Patients with Type 2 Diabetes Mellitus. Yangtze Medicine, 2, 129-145. https://doi.org/10.4236/ym.2018.23014

Received: May 5, 2018

Accepted: August 27, 2018

Published: August 30, 2018

Copyright $\odot 2018$ by authors and Scientific Research Publishing Inc. This work is licensed under the Creative Commons Attribution International License (CC BY 4.0).

http://creativecommons.org/licenses/by/4.0/

\begin{abstract}
Objective: To evaluate the safety of dapagliflozin for Type 2 Diabetes Mellitus (T2DM). Methods: A systematic search of Pubmed, Embase, Cochrance Library, Web of Science, CNKI, Wanfang Data and VIP database for randomized controlled trials (RCTs) comparing dapagliflozin with placebo was performed up to February 2018. The index words included dapagliflozin, type 2 diabetes mellitus and randomized controlled trial. Results: A total of 19 RCTs involving 7704 participants were incorporated into the study. Compared with placebo, dapagliflozin did not increase the risk of hypoglycemia $[\mathrm{OR}=1.14,95 \% \mathrm{CI}(0.95,1.36), \mathrm{P}=0.17]$ and hypotension $[\mathrm{OR}=1.43,95 \% \mathrm{CI}$ $(0.94,2.17), \mathrm{P}=0.10]$, but significantly increased the incidences of renal adverse events [OR $=1.57,95 \% \mathrm{CI}(1.17,2.09), \mathrm{P}=0.002]$, genital tract infection $[\mathrm{OR}=3.65,95 \% \mathrm{CI}(2.93,4.56), \mathrm{P}<0.00001]$ and urinary tract infection [OR $=1.36,95 \% \mathrm{CI}(1.15,1.61), \mathrm{P}=0.0004]$ and related doses. Conclusions: Generally, dapagliflozin had no risk of hypoglycemia and hypotension in patients with T2DM, but there were risks of renal adverse events and urogenital tract infection. Due to the limitations of this study, larger samples and RCTs with long-term follow-up are needed for further verification.
\end{abstract}

\section{Keywords}

Dapagliflozin, Type 2 Diabetes Mellitus, Randomized Controlled Trial, Meta-Analysis

\section{Introduction}

As a common disease in China, the incidence of T2DM has been increasing year by year, and drug therapy is the main method of treatment. Sodium glucose co-transporter 2 (SGLT-2), as the most important glucose transporter in the kidney, is responsible for the reabsorption of $90 \%$ glucose in the kidney [1]. The 
SGLT-2 inhibitor, dapagliflozin is a novel oral hypoglycemic drug for the treatment of T2DM, which is independent from islet $\beta$-cell function and insulin sensitivity. It functions by increasing renal clearance of glucose, attenuating renal reabsorption of glucose and excreting excess glucose from the urine, thereby reducing blood glucose [2]. Studies have shown that dapagliflozin has multiple benefits such as reduction in blood glucose, weight and blood pressure. However, there is also risk for urogenital tract infections [3]. In this study, the safety is further analyzed by retrieving RCTs of dapagliflozin versus placebo through meta-analysis.

\section{Data and Methods}

\subsection{Criteria for Inclusion and Exclusion of Literature}

Inclusion criteria:

1) Research type: Included in the study were clinical RCTs of different doses of dapaglilfozin versus placebo in the treatment of T2DM officially published before February 2018; full text is available; languages are limited to Chinese and English.

2) Research object: Age $>18$ years, in accordance with the diagnostic criteria for T2DM (WHO Committee of Experts on Diabetes 1999 diagnosis and classification criteria), in accordance with the dapagliflozin guidelines.

3) Interventions: The experimental group was treated with dapagliflozan alone, regardless of dose size; placebo in the control group; or on the basis of other similar interventions.

Exclusion criteria:

Treatment observation time $<12$ weeks, type 1 and special types of diabetes, review, non-RCT and so on.

\subsection{Evaluation Index}

Safety related indicators: hypoglycemia events, hypotension events, adverse renal reactions, genital tract infections, urinary tract infections.

\subsection{Search Strategy}

The search strategy follows the Preferred Reporting Items for Systematic Reviews and Meta-Analyses (PRISMA) statement [4]. Relevant studies for the analysis were selected by searching PubMed, Embase, Cochrance Library, Web of Science, CNKI, Wanfang Data and VIP database up to the end of February 2018. Taking Pubmed database as an example, subject words and free words are combined to search, and the retrieval scheme is as follows: \#1 AND \#2 AND \#3.

\#1 ((dapagliflozin) OR forxiga). \#2 Noninsulin-Dependent) OR Diabetes Mellitus, Ketosis-Resistant) OR Diabetes Mellitus, Ketosis Resistant) OR Ketosis-Resistant Diabetes Mellitus) OR Diabetes Mellitus, Non Insulin Dependent) OR Diabetes Mellitus, Non-Insulin-Dependent) OR Non-Insulin-Dependent Diabetes Mellitus) OR Diabetes Mellitus, Stable) 
OR Stable Diabetes Mellitus) OR Diabetes Mellitus, Type II) OR NIDDM) OR Diabetes Mellitus, Noninsulin Dependent) OR Diabetes Mellitus, Maturity-Onset) OR Diabetes Mellitus, Maturity Onset) OR Maturity-Onset Diabetes Mellitus) OR Maturity Onset Diabetes Mellitus) OR MODY) OR Diabetes Mellitus, Slow-Onset) OR Diabetes Mellitus, Slow Onset) OR Slow-Onset Diabetes Mellitus) OR Type 2 Diabetes Mellitus) OR Noninsulin-Dependent Diabetes Mellitus) OR Noninsulin Dependent Diabetes Mellitus) OR Maturity-Onset Diabetes) OR Diabetes, Maturity-Onset) OR Maturity Onset Diabetes) OR Type 2 Diabetes) OR Diabetes, Type 2) OR Diabetes Mellitus, Adult-Onset) OR Adult-Onset Diabetes Mellitus) OR Diabetes Mellitus, Adult Onset). \#3 (((randomized controlled trial) OR randomized) OR placebo).

\subsection{Data Extraction}

Data were independently abstracted by the two principal reviewers and any discrepancies were resolved by consensus. The data extracted included title, author, and year of publication, country, intervention measures, sample size, patient age, treatment time, main outcome indicators and intentionality analysis.

\subsection{Quality Assessment}

Jadad scoring criteria were used [5], including 4 items: random method, assignment concealment, blind method and withdrawal. The RCT score was 1 - 7, 1 - 3 as low quality and $4-7$ as high quality.

\subsection{Data Analysis}

Outcomes were pooled using Review Manager 5.3 software. Dichotomous data were presented as odds ratio (OR) or risk ratio (RR). All results were estimated from each study with $95 \%$ confidence intervals (CIs). Heterogeneity was assessed using the $\mathrm{I}^{2}$ statistic. If $\mathrm{P}>0.1, \mathrm{I}^{2}<50 \%$, a fixed-effect model with the Mantel-Haenszel method was used; otherwise, the random-effect model was adopted. Subgroup analysis was performed according to the different doses of dapagliflozin ( $1 \mathrm{mg}, 2.5 \mathrm{mg}, 5 \mathrm{mg}, 10 \mathrm{mg}$ and $20 \mathrm{mg}$ ). Funnel plot was adopted to evaluate the publication bias. The bilaterally symmetric graph indicates a low risk of publication bias. Otherwise, the risk of publication bias may exist.

\section{Results}

\subsection{The Basic Characteristics and Process of Inclusion Study}

A total of 19 RCTs involving 7704 participants was incorporated into the study, including 5304 cases of dapagliflozin group and 2400 cases of the placebo group. All 19 RCTs were performed from 2009 to 2016, and the follow-up duration was $\geq 12$ weeks. Baseline data for both groups were described in all trials, and there was no statistical difference in general between the dapagliflozin and placebo groups before treatment $(\mathrm{P}>0.05)$. The screening process is displayed in Figure 1. The basic information contained in the study is shown in Table 1 . 


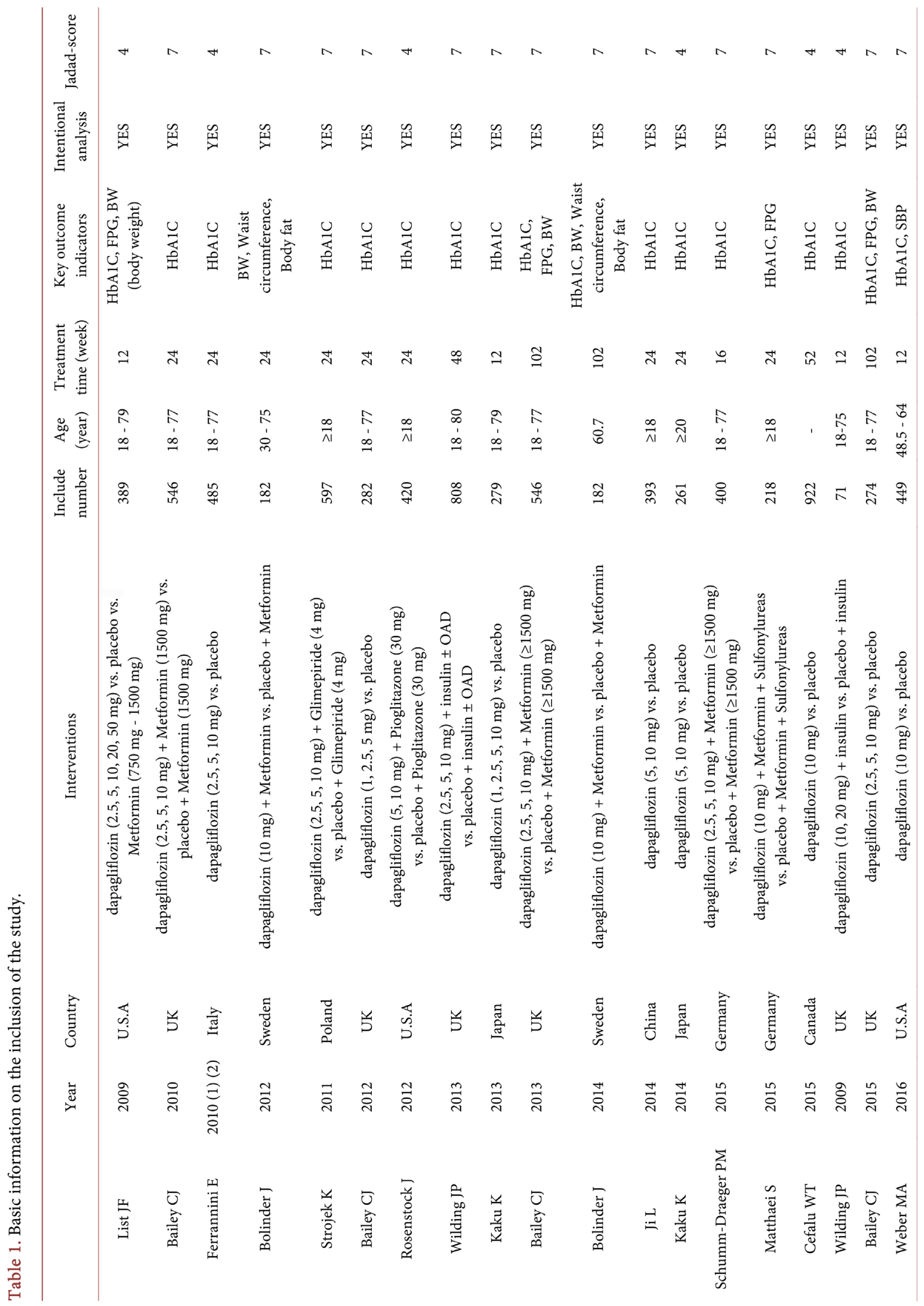




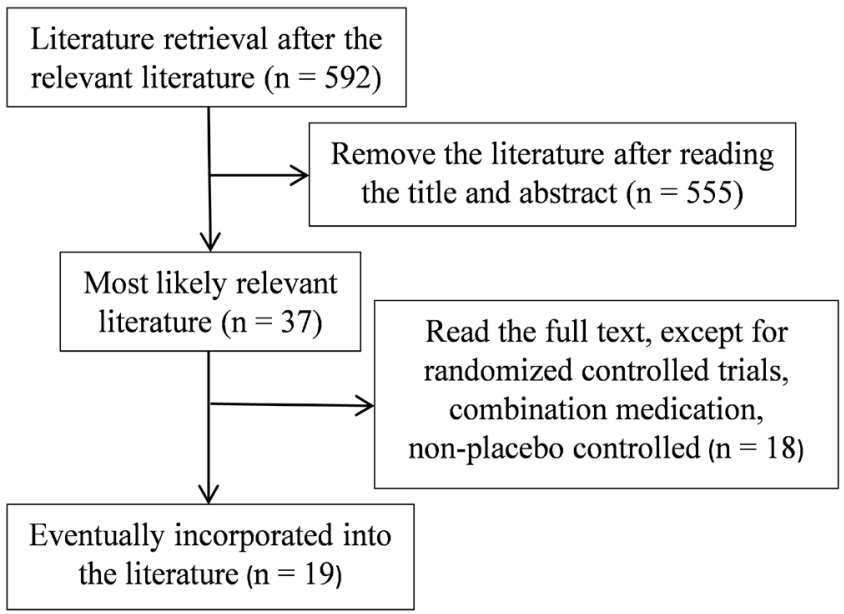

Figure 1. Screening flow chart for inclusion studies.

\subsection{Risk Assessment of Bias Included in the Study}

Two reviewers independently applied the Cochrane Risk of Bias tool to assess the risk of bias in the RCTs. The following methodological features relevant to the minimization of bias were assessed: randomization, allocation concealment, blinding of participants and personnel, blinding of outcome assessment, incomplete outcome data, selective reporting, and other bias. The following judgments were used: low risk, high risk, or unclear risk (either lack of information or uncertainty regarding the potential for bias). The bias risk assessment is shown in Figure 2.

\section{Results of Meta-Analysis}

\subsection{Hypoglycemia Event}

A total of 19 studies were implemented [3] [6]-[23] to compare hypoglycemia events. Heterogeneity was detected in study subgroups $(2.5 \mathrm{mg}, 5 \mathrm{mg}, 10 \mathrm{mg}, 20$ $\mathrm{mg})$, indicating that there was no statistically significant heterogeneity $\left(\mathrm{I}^{2}=0 \%\right.$, $\mathrm{P}=0.96),\left(\mathrm{I}^{2}=0 \%, \mathrm{P}=0.67\right),\left(\mathrm{I}^{2}=0 \%, \mathrm{P}=0.70\right),\left(\mathrm{I}^{2}=0 \%, \mathrm{P}=0.89\right)$ between studies included in the analysis (Figure 3 ). Therefore, the M-H fixation effect model was used for meta-analysis. Funnel plot of publication bias evaluation is presented in Figure 4. There was no risk of publication bias among subgroups in the study. No significant differences were observed in the comparison results of dapagliflozin with placebo in the incidence of hypoglycemia event $[\mathrm{OR}=1.14$, 95\% CI $(0.95,1.36), \mathrm{P}=0.17]$, as shown in Figure 3.

\subsection{Hypotension Event}

A total of 13 studies were implemented [6]-[11] [13] [15] [16] [18] [19] [20] [22] to compare hypotension events. Heterogeneity was detected in the study subgroups $(2.5 \mathrm{mg}, 5 \mathrm{mg}, 10 \mathrm{mg}$ ), indicating that there was no statistically significant heterogeneity $\left(\mathrm{I}^{2}=0 \%, \mathrm{P}=0.60\right),\left(\mathrm{I}^{2}=0 \%, \mathrm{P}=0.85\right),\left(\mathrm{I}^{2}=0 \%, \mathrm{P}=0.79\right)$ between studies included in the analysis (Figure 5 ). Therefore, the $\mathrm{M}-\mathrm{H}$ fixation 


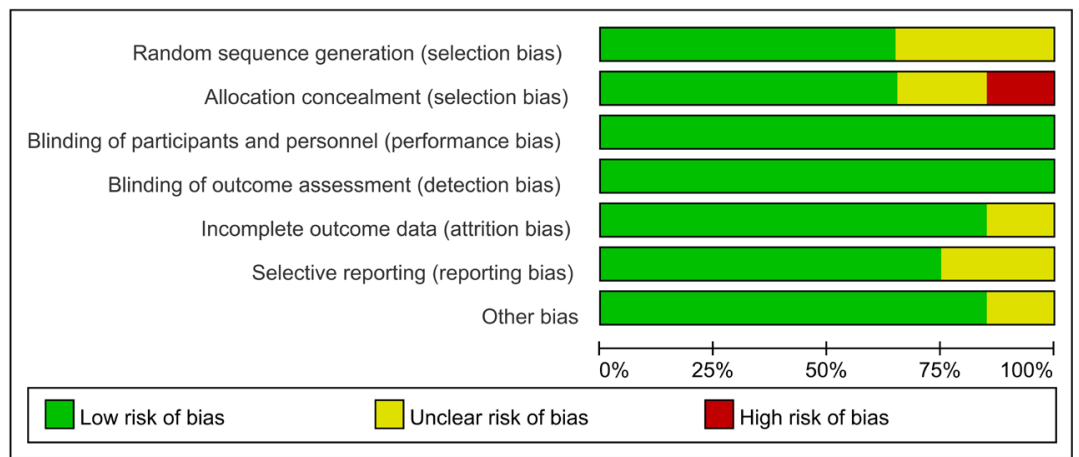

(a)

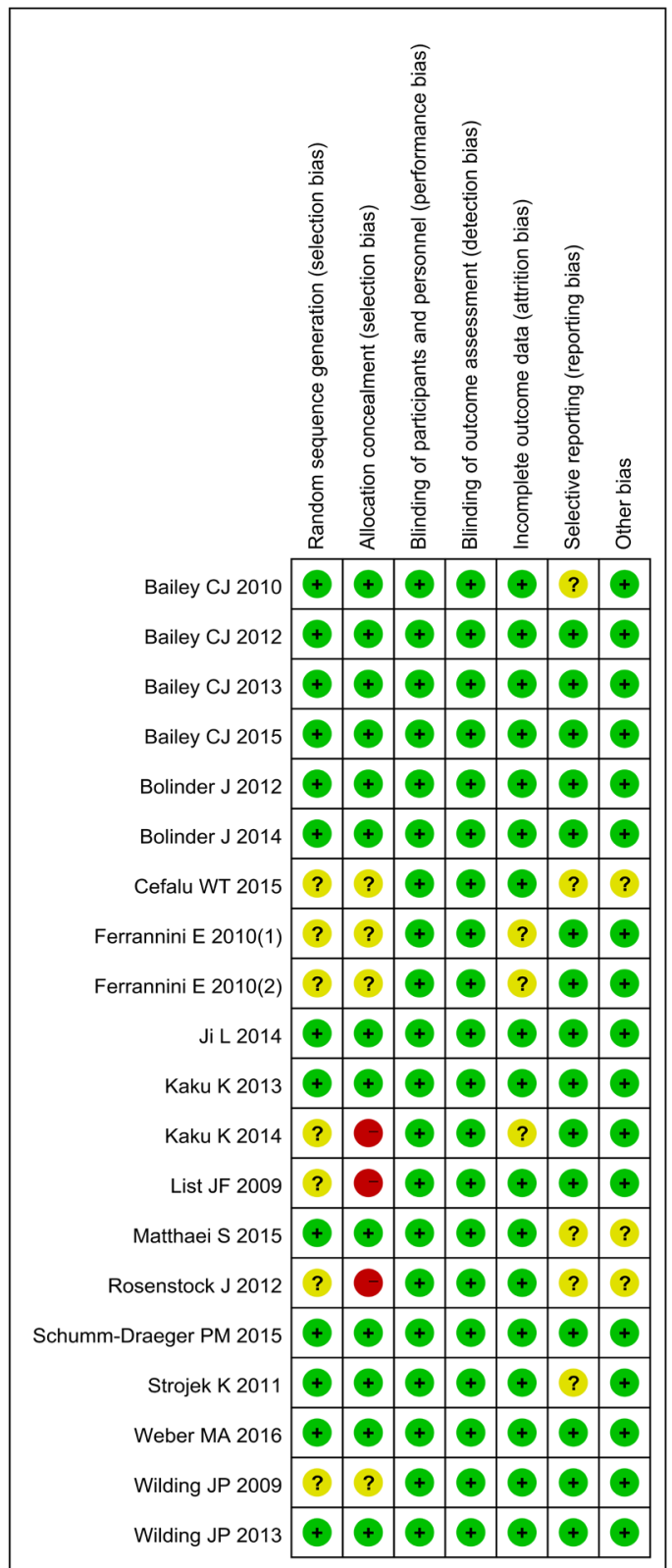

(b)

Figure 2. Bias risk assessment charts. 


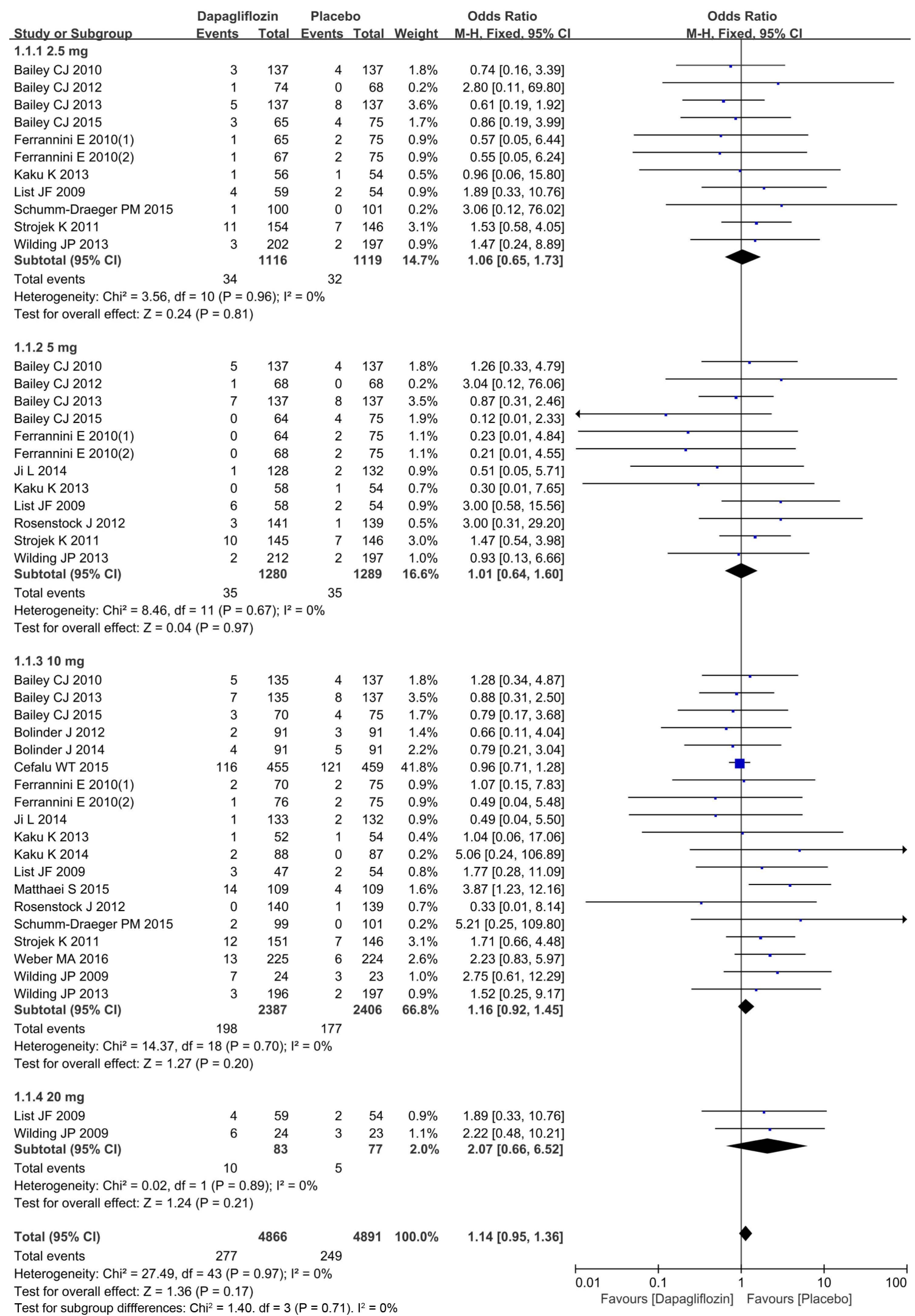

Figure 3. Comparison of dapagliflozin versus placebo for hypoglycaemic events in patients with T2DM in different dose subgroups. 


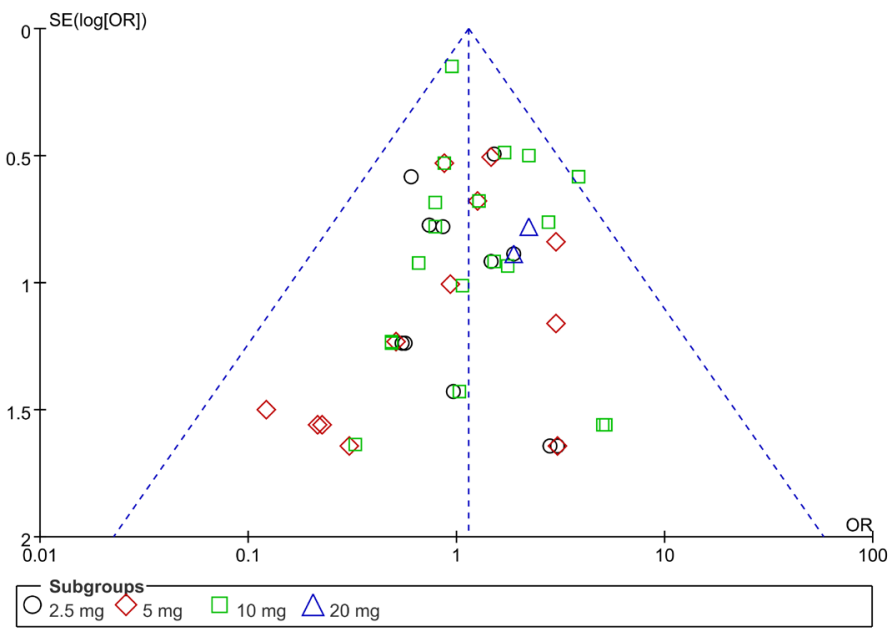

Figure 4. Funnel plot for publication bias evalution of dapagliflozin versus placebo for hypoglycemia events in patients with T2DM in different dose subgroups.

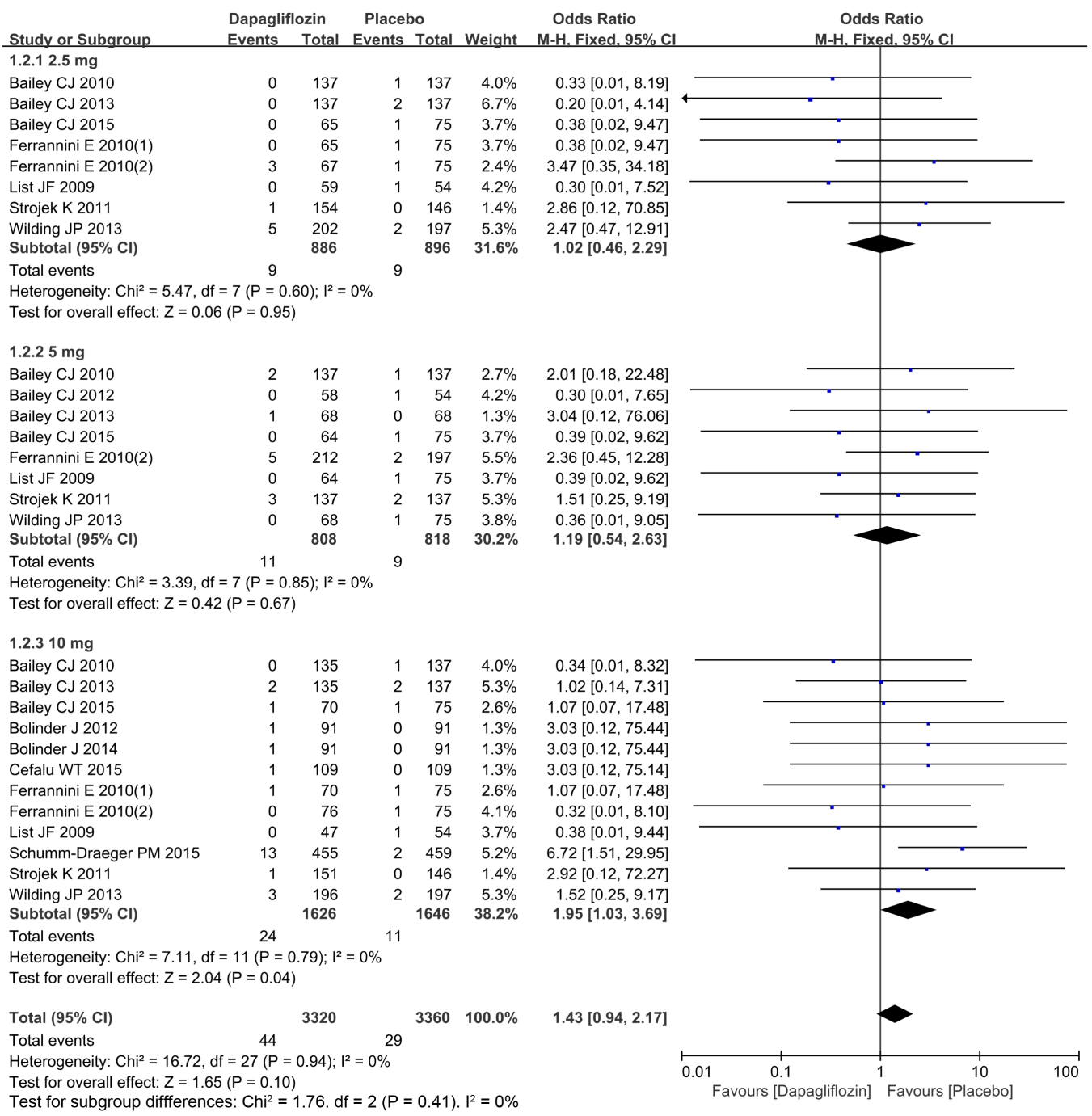

Figure 5. Comparison of dapagliflozin versus placebo for hypotention events in patients with T2DM in different dose subgroups. 
effect model was used for meta-analysis. Funnel plot of publication bias evaluation is presented in Figure 6. There was no risk of publication bias among subgroups in the study. No significant differences were observed in the comparison results of dapagliflozin with placebo in the incidence of hypotension event [OR $=1.43,95 \% \mathrm{CI}(0.94,2.17), \mathrm{P}=0.10]$, as shown in Figure 5 .

\subsection{Renal Adverse Event}

A total of 13 studies were implemented [3] [9] [10] [12] [13] [15]-[20] [22] [23] to compare renal adverse events. Heterogeneity was detected in study subgroups $(2.5 \mathrm{mg}, 5 \mathrm{mg}, 10 \mathrm{mg})$, indicating that there was no statistically significant heterogeneity $\left(\mathrm{I}^{2}=0 \%, \mathrm{P}=0.42\right),\left(\mathrm{I}^{2}=0 \%, \mathrm{P}=0.85\right),\left(\mathrm{I}^{2}=0 \%, \mathrm{P}=0.89\right)$ between studies included in the analysis (Figure 7). Therefore, the $\mathrm{M}-\mathrm{H}$ fixation effect model was used for meta-analysis. Funnel plot of publication bias evaluation is presented in Figure 8. There was no risk of publication bias among subgroups in the study. Compared with placebo, dapagliflozin (10 mg subgroup) significantly increased the incidences of renal adverse events $[\mathrm{OR}=1.57,95 \% \mathrm{CI}(1.17,2.09)$, $\mathrm{P}=0.002]$, as shown in Figure 7.

\subsection{Genital Tract Infections}

A total of 19 studies were implemented [3] [6]-[23] to compare genital tract infections. Heterogeneity was detected in the study subgroups $(2.5 \mathrm{mg}, 5 \mathrm{mg}, 10$ $\mathrm{mg}, 20 \mathrm{mg}$ ), indicating that there was no statistically significant heterogeneity $\left(\mathrm{I}^{2}=0 \%, \mathrm{P}=0.81\right),\left(\mathrm{I}^{2}=0 \%, \mathrm{P}=0.98\right),\left(\mathrm{I}^{2}=0 \%, \mathrm{P}=0.64\right),\left(\mathrm{I}^{2}=0 \%, \mathrm{P}=0.82\right)$ between studies included in the analysis (Figure 9). Therefore, the M-H fixation effect model was used for meta-analysis. Funnel plot of publication bias evaluation is presented in Figure 10. There was no risk of publication bias among

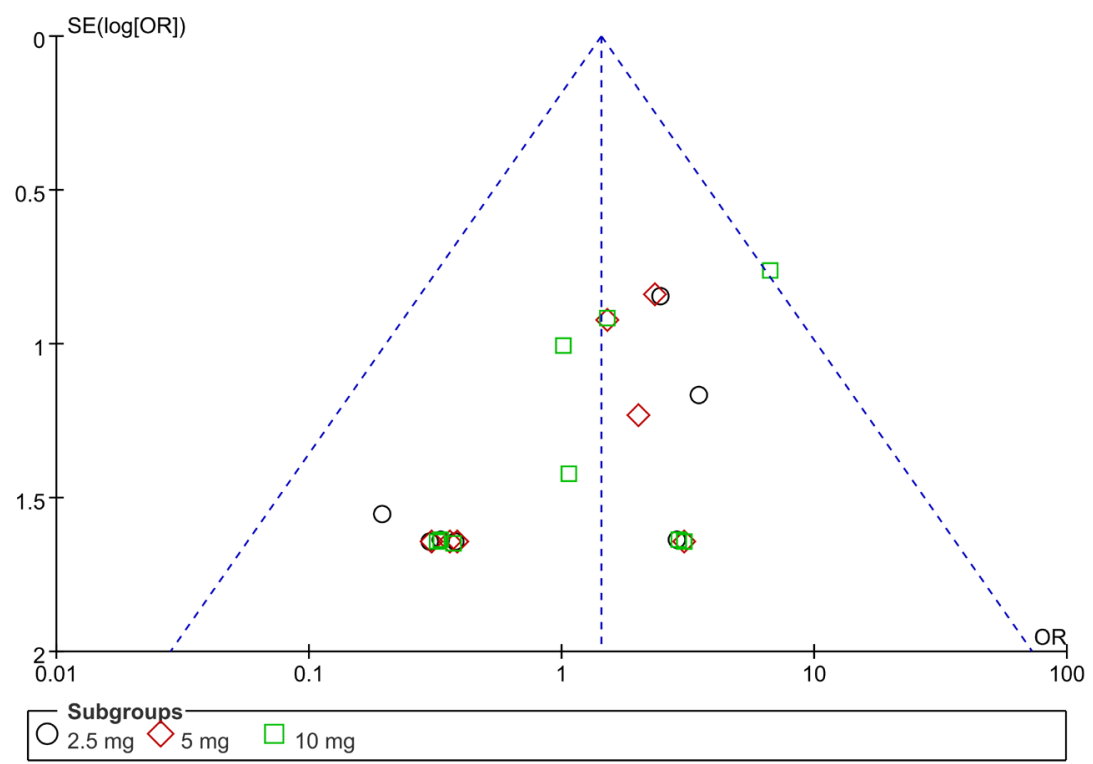

Figure 6. Funnel plot for publication bias evalution of dapagliflozin versus placebo for hypotention events in patients with T2DM in different dose subgroups. 


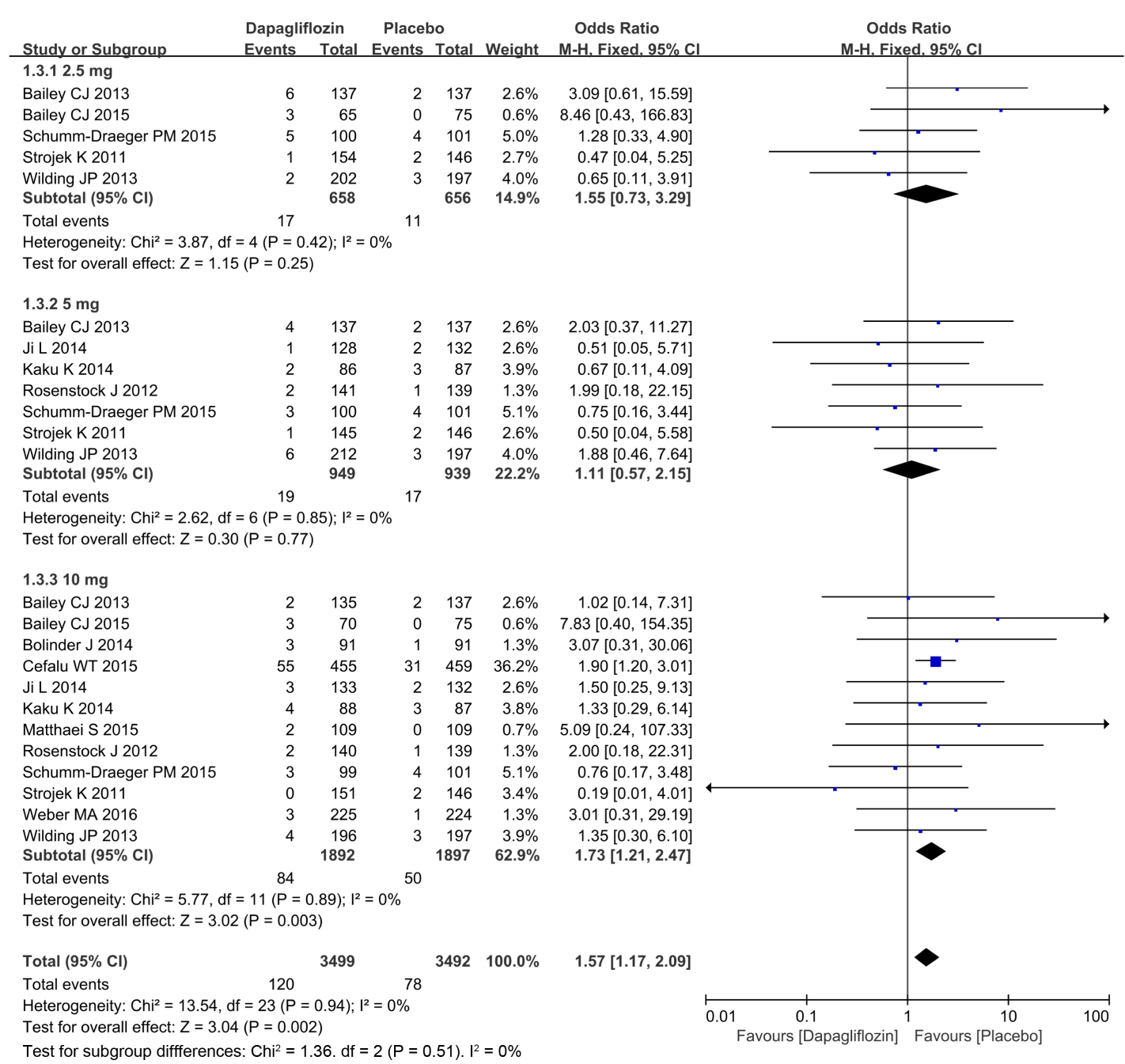

Figure 7. Comparison of dapagliflozin versus placebo for renal adverse events in patients with T2DM in different dose subgroups.

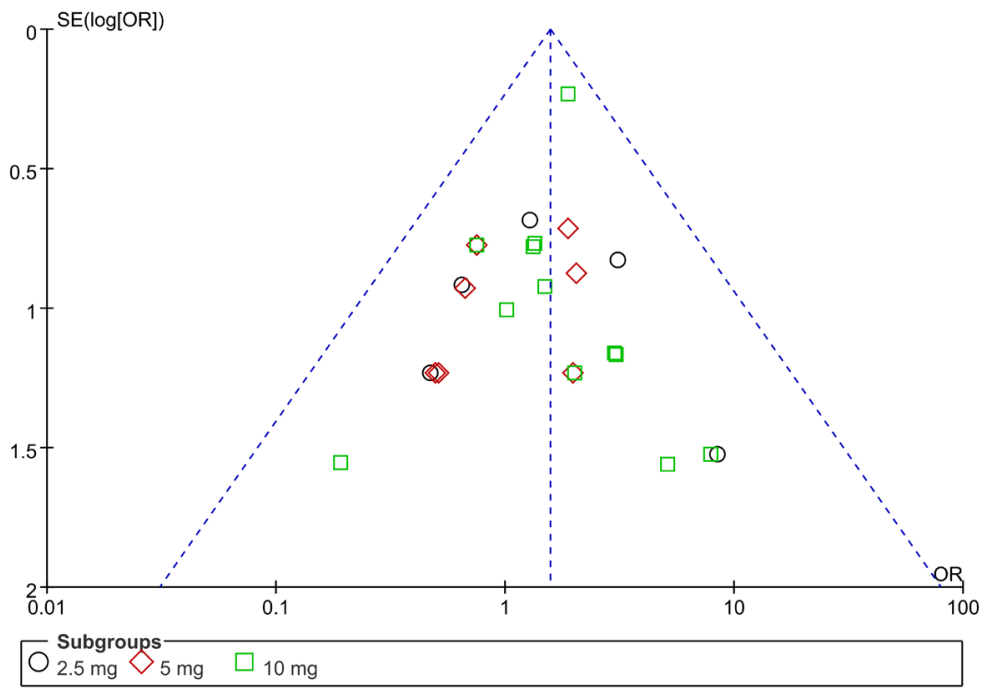

Figure 8. Funnel plot for publication bias evalution of dapagliflozin versus placebo for renal adverse events in patients with T2DM in different dose subgroups. 


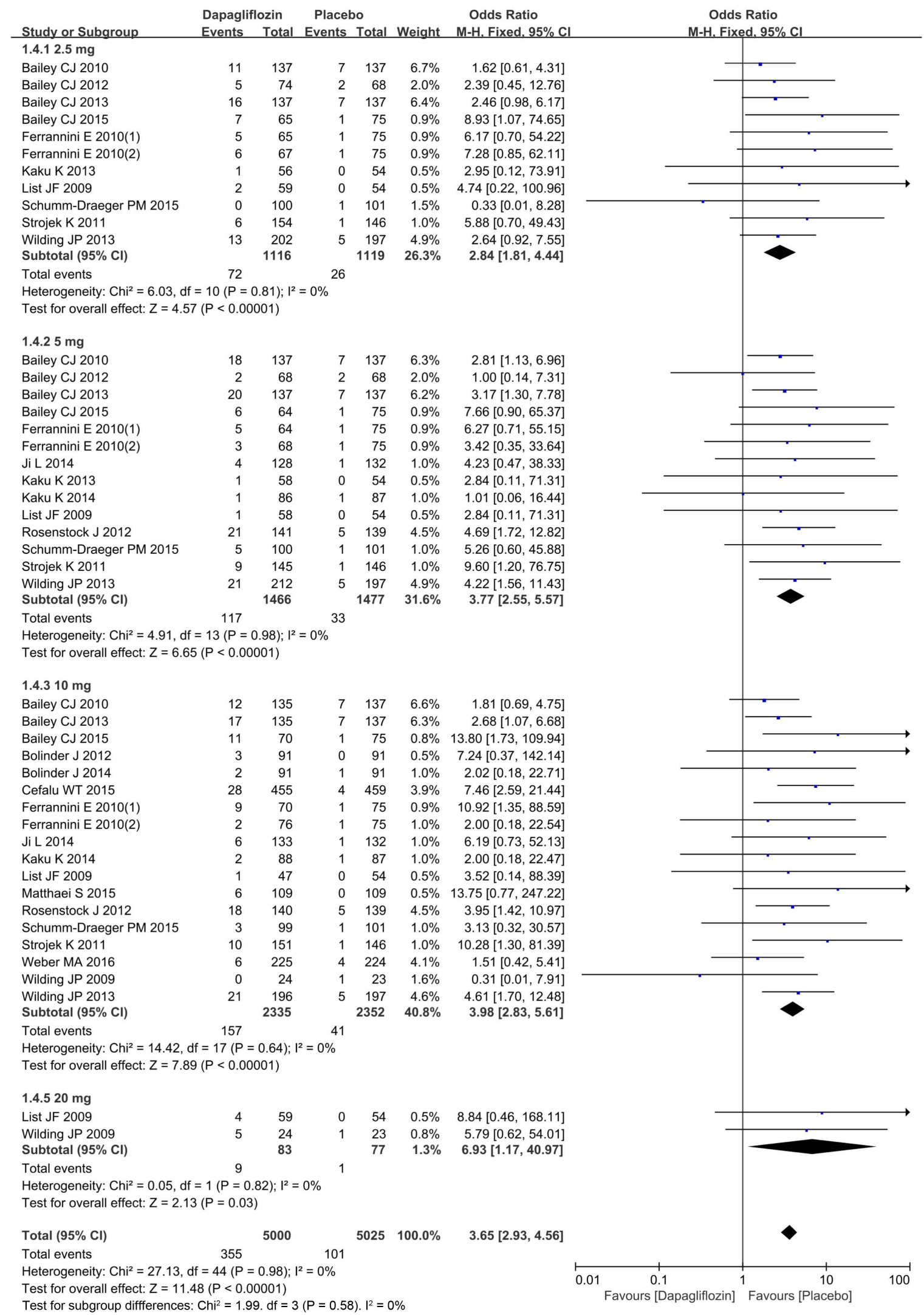

Figure 9. Comparison of dapagliflozin versus placebo for genital tract infections in patients with T2DM in different dose subgroups. 


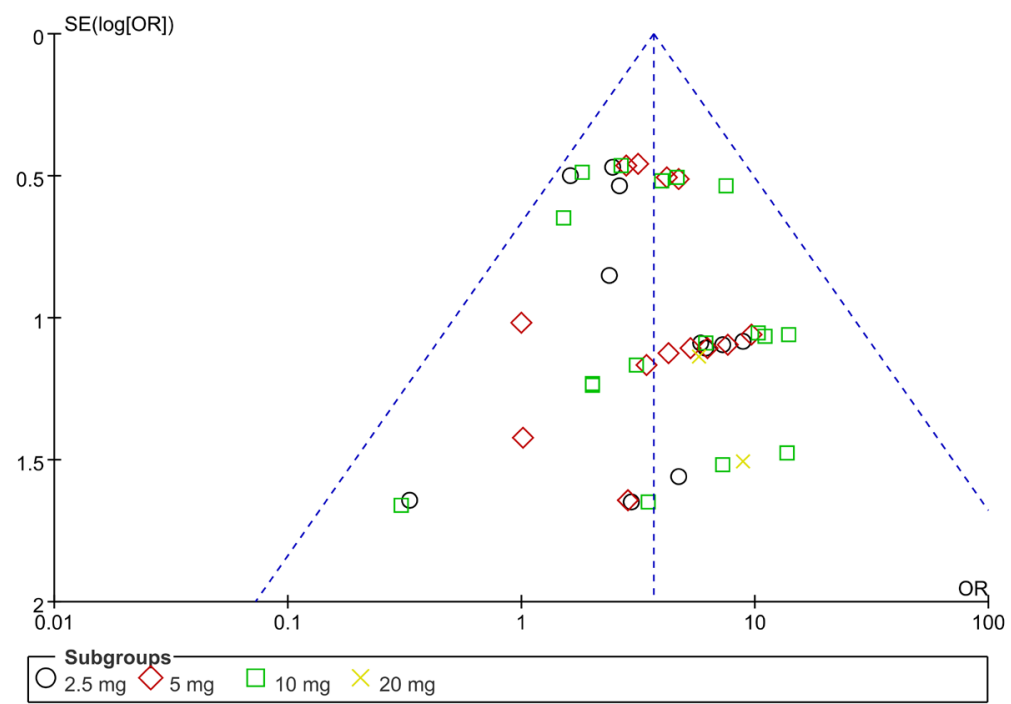

Figure 10. Funnel plot for publication bias evalution of dapagliflozin versus placebo for genital tract infections in patients with T2DM in different dose subgroups.

subgroups in the study. Compared with placebo, dapagliflozin $(2.5 \mathrm{mg}, 5 \mathrm{mg}, 10$ $\mathrm{mg}, 20 \mathrm{mg}$ subgroup) significantly increased the incidences of genital tract infections $[\mathrm{OR}=3.65,95 \% \mathrm{CI}(2.93,4.56), \mathrm{P}<0.00001]$, as shown in Figure 9.

\subsection{Urinary Tract Infections}

A total of 19 studies were included [3] [6]-[23] to compare urinary tract infections. Heterogeneity was detected in the study subgroups $(1 \mathrm{mg}, 2.5 \mathrm{mg}, 5 \mathrm{mg}$, $10 \mathrm{mg}, 20 \mathrm{mg}$ ), indicating that there was no statistically significant heterogeneity $\left(\mathrm{I}^{2}=0 \%, \mathrm{P}=0.53\right),\left(\mathrm{I}^{2}=0 \%, \mathrm{P}=0.86\right),\left(\mathrm{I}^{2}=0 \%, \mathrm{P}=0.77\right),\left(\mathrm{I}^{2}=0 \%, \mathrm{P}=0.97\right),\left(\mathrm{I}^{2}\right.$ $=0 \%, \mathrm{P}=0.88$ ) between studies included in the analysis (Figure 11). Therefore, the $\mathrm{M}-\mathrm{H}$ fixation effect model was used for meta-analysis. Funnel plot of publication bias evaluation is presented in Figure 12. There was no risk of publication bias among subgroups in the study. Compared with placebo, dapagliflozin (5 $\mathrm{mg}, 10 \mathrm{mg}$ subgroup) significantly increased the incidences of urinary tract infections $[\mathrm{OR}=1.36,95 \% \mathrm{CI}(1.15,1.61), \mathrm{P}=0.0004]$, as shown in Figure 11.

\section{Discussion}

The meta-analysis suggests that dapagliflozin had no difference in the risk of hypoglycemia and hypotension events compared with placebo, and was safer than sulfonylurea, insulin and other hypoglycemic agents with a higher risk of hypoglycemia. Studies have illustrated that Chinese adults with type 2 diabetes were more complicated due to hypertension and other cardiovascular risk factors [24]. Dapagliflozin can reduce blood glucose while reducing blood pressure and weight [25] [26], improve oxidative stress and endothelial function and reduce arteriosclerosis [27] [28], with potential cardiovascular benefits [29]. Multiple studies on the effects of renal function proved that the eGFR and urinary albumin-creatinine ratio were not significantly changed in the dapagliflozin 


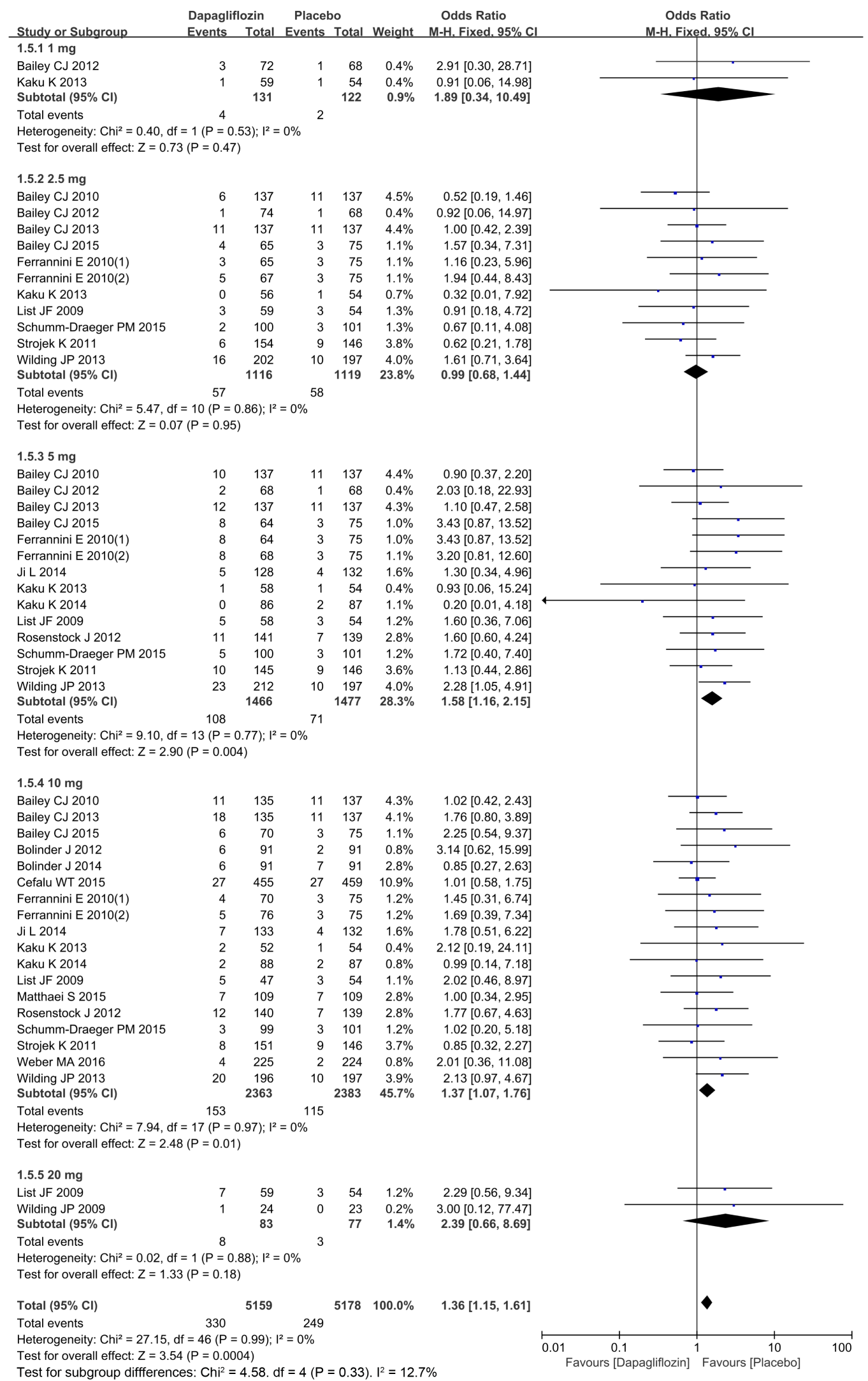

Figure 11. Comparison of dapagliflozin versus placebo for urinary tract infections in patients with T2DM in different dose subgroups. 


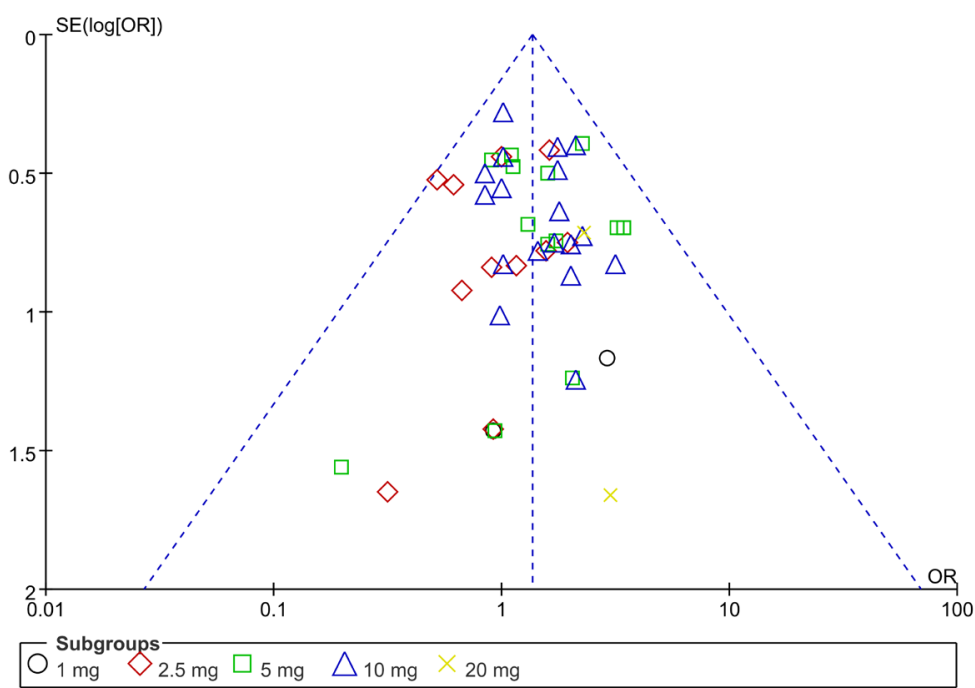

Figure 12. Funnel plot for publication bias evalution of dapagliflozin versus placebo for urinary tract infections in patients with T2DM in different dose subgroups.

treatment group by contrast with the placebo group in patients with T2DM [30] [31] [32]. The higher risk of renal adverse events than placebo was associated primarily with the study by Cefalu WT et al., which involved a population with a history of type 2 diabetes mellitus associated with pre-cardiovascular disease, hypertension. They defined renal adverse events as renal damage, acute renal failure, decreased creatinine clearance, increased serum creatinine, and decreased glomerular filtration rate, renal dysfunction and other serological indicators. For patients with stage $3 \mathrm{CKD}$ or greater, dapagliflozin either requires dose adjustment or is contraindicated [33].

The 19 trials involved in this study are qualified English literature, all of which are single-center or multi-center studies with high methodological quality. There is no significant publication bias in all the literature, so the conclusions are reliable. However, a variety of factors such as the inclusion of subjects, patients with type 2 diabetes, disease severity, duration of illness, combination of other diseases with dapagliflozin dose and medication time may increase the clinical heterogeneity of the study.

\section{Conflicts of Interest}

The authors declare no conflicts of interest regarding the publication of this paper.

\section{References}

[1] Lee, Y.J. and Han, H.J. (2007) Regulatory Mechanisms of $\mathrm{Na(+)/Glucose} \mathrm{Cotrans-}$ porters in Renal Proximal Tubule Cells. Kidney International, 106, S27-S35. https://doi.org/10.1038/sj.ki.5002383

[2] Gerich, J.E. (2010) Role of the Kidney in Normal Glucose Homeostasis and in the Hyperglycaemia of Diabetes Mellitus: Therapeutic Implications. Diabetic Medicine, 27, 136-142. https://doi.org/10.1111/j.1464-5491.2009.02894.x

[3] Ji, L., Ma, J., Li, H., et al. (2014) Dapagliflozin as Monotherapy in Drug-Naive Asian 
Patients with Type 2 Diabetes Mellitus: A Randomized, Blinded, Prospective Phase III Study. Clinical Therapeutics, 36, 84-100.

https://doi.org/10.1016/j.clinthera.2013.11.002

[4] Knobloch, K., Yoon, U. and Vogt, P.M. (2011) Preferred Reporting Items for Systematic Reviews and Meta-Analyses (PRISMA) Statement and Publication Bias. Journal of Cranio-Maxillo-Facial Surgery, 39, 91-92.

https://doi.org/10.1016/j.jcms.2010.11.001

[5] Jadad, A.R., Moore, R.A., Carroll, D., et al. (1996) Assessing the Quality of Reports of Randomized Clinical Trials: Is Blinding Necessary? Controlled Clinical Trials, 17, 1-12. https://doi.org/10.1016/0197-2456(95)00134-4

[6] List, J.F., Woo, V., Morales, E., et al. (2009) Sodium-Glucose Cotransport Inhibition with Dapagliflozin in Type 2 Diabetes. Diabetes Care, 32, 650-657.

https://doi.org/10.2337/dc08-1863

[7] Bailey, C.J., Gross, J.L., Pieters, A., et al. (2010) Effect of Dapagliflozin in Patients with Type 2 Diabetes Who Have Inadequate Glycaemic Control with Metformin: A Randomised, Double-Blind, Placebo-Controlled Trial. Lancet, 375, 2223-2233. https://doi.org/10.1016/S0140-6736(10)60407-2

[8] Ferrannini, E., Ramos, S.J., Salsali, A., et al. (2010) Dapagliflozin Monotherapy in Type 2 Diabetic Patients with Inadequate Glycemic Control by Diet and Exercise: A Randomized, Double-Blind, Placebo-Controlled, Phase 3 Trial. Diabetes Care, 33, 2217-2224. https://doi.org/10.2337/dc10-0612

[9] Bolinder, J., Ljunggren, Ö., Kullberg, J., et al. (2012) Effects of Dapagliflozin on Body Weight, Total Fat Mass, and Regional Adipose Tissue Distribution in Patients with Type 2 Diabetes Mellitus with Inadequate Glycemic Control on Metformin. The Journal of Clinical Endocrinology \& Metabolism, 97, 1020-1031. https://doi.org/10.1210/jc.2011-2260

[10] Strojek, K., Yoon, K.H., Hruba, V., et al. (2011) Effect of Dapagliflozin in Patients with Type 2 Diabetes Who Have Inadequate Glycaemic Control with Glimepiride: A Randomized, 24-Week, Double-Blind, Placebo-Controlled Trial. Diabetes Obesity and Metabolism, 13, 928-938. https://doi.org/10.1111/j.1463-1326.2011.01434.x

[11] Bailey, C.J., Iqbal, N., T’joen, C., et al. (2012) Dapagliflozin Monotherapy in Drug-Naïve Patients with Diabetes: A Randomized-Controlled Trial of Low-Dose Range. Diabetes Obesity and Metabolism, 14, 951-959.

https://doi.org/10.1111/j.1463-1326.2012.01659.x

[12] Rosenstock, J., Vico, M., Wei, L., et al. (2012) Effects of Dapagliflozin, an SGLT2 Inhibitor, on HbA1c, Body Weight, and Hypoglycemia Risk in Patients with Type 2 Diabetes Inadequately Controlled on Pioglitazone Monotherapy. Diabetes Care, 35, 1473-1478. https://doi.org/10.2337/dc11-1693

[13] Wilding, J.P., Woo, V., Soler, N.G., et al. (2013) Long-Term Efficacy of Dapagliflozin in Patients with Type 2 Diabetes Mellitus Receiving High Doses of Insulin. Dtsch Med Wochenschr, 138, S27-S38. https://doi.org/10.1055/s-0032-1305284

[14] Kaku, K., Inoue, S., Matsuoka, O., et al. (2013) Efficacy and Safety of Dapagliflozin as a Monotherapy for Type 2 Diabetes Mellitus in Japanese Patients with Inadequate Glycaemic Control: A Phase II Multicentre, Randomized, Double-Blind, Placebo-Controlled Trial. Diabetes Obesity and Metabolism, 15, 432-440. https://doi.org/10.1111/dom.12047

[15] Bailey, C.J., Gross, J.L., Hennicken, D., et al. (2013) Dapagliflozin Add-On to Metformin in Type 2 Diabetes Inadequately Controlled with Metformin. A Randomized, Double-Blind, Placebo-Controlled 102-Week Trial. BMC Medicine, 11, 43. 
https://bmcmedicine.biomedcentral.com/articles/10.1186/1741-7015-11-43

[16] Bolinder, J., Ljunggren, Ö., Johansson, L., et al. (2014) Dapagliflozin Maintains Glycaemic Control While Reducing Weight and Body Fat Mass over 2 Years in Patients with Type 2 Diabetes Mellitus Inadequately Controlled on Metformin. Diabetes, Obesity and Metabolism, 16, 159-169. https://doi.org/10.1111/dom.12189

[17] Kaku, K., Kiyosue, A., Inoue, S., et al. (2014) Efficacy and Safety of Dapagliflozin Monotherapy in Japanese Patients with Type 2 Diabetes Inadequately Controlled by Diet and Exercise. Diabetes, Obesity and Metabolism, 16, 1102-1110. https://doi.org/10.1111/dom.12325

[18] Schumm-Draeger, P.M., Burgess, L., Korányi, L., et al. (2015) Twice-Daily Dapagliflozin Co-Administered with Metformin in Type 2 Diabetes: A 16-Week Randomized, Placebo-Controlled Clinical Trial. Diabetes, Obesity and Metabolism, 17, 42-51. https://doi.org/10.1111/dom.12387

[19] Matthaei, S., Bowering, K., Rohwedder, K., et al. (2015) Dapagliflozin Improves Glycemic Control and Reduces Body Weight as Add-On Therapy to Metformin plus Sulfonylurea: A 24-Week Randomized, Double-Blind Clinical Trial. Diabetes Care, 38, 365-372. https://doi.org/10.2337/dc14-0666

[20] Cefalu, W.T., Leiter, L.A., de Bruin, T.W., et al. (2015) Dapagliflozin's Effects on Glycemia and Cardiovascular Risk Factors in High-Risk Patients with Type 2 Diabetes: A 24-Week, Multicenter, Randomized, Double-Blind, Placebo-Controlled Study with a 28-Week Extension. Diabetes Care, 38, 1218-1227. https://doi.org/10.2337/dc14-0315

[21] Wilding, J.P., Norwood, P., T'joen, C., et al. (2009) A Study of Dapagliflozin in Patients with Type 2 Diabetes Receiving High Doses of Insulin plus Insulin Sensitizers: Applicability of a Novel Insulin-Independent Treatment. Diabetes Care, 32, 1656-1662. https://doi.org/10.2337/dc09-0517

[22] Bailey, C.J., Morales Villegas, E.C., Woo, V., et al. (2015) Efficacy and Safety of Dapagliflozin Monotherapy in People with Type 2 Diabetes: A Randomized Double-Blind Placebo-Controlled 102-Week Trial. Diabetic Medicine, 32, 531-541. https://doi.org/10.1111/dme.12624

[23] Weber, M.A., Mansfield, T.A., Cain, V.A., et al. (2016) Blood Pressure and Glycaemic Effects of Dapagliflozin versus Placebo in Patients with Type 2 Diabetes on Combination Antihypertensive Therapy: A Randomised, Double-Blind, Placebo-Controlled, Phase 3 Study. The Lancet Diabetes \& Endocrinology, 4, 211-220. https://doi.org/10.1016/S2213-8587(15)00417-9

[24] Ji, L.N., Hu, D., Pan, C., et al. (2013) Primacy of the 3B Approach to Control Risk Factors for Cardiovascular Disease in Type 2 Diabetes Patients. American Journal of Medicine, 126, 925.e11-22. https://doi.org/10.1016/j.amjmed.2013.02.035

[25] Zhang, M., Zhang, L., Wu, B., et al. (2014) Dapagliflozin Treatment for Type 2 Diabetes: A Systematic Review and Meta-Analysis of Randomized Controlled Trials. Diabetes/Metabolism Research and Reviews, 30, 204-221. https://doi.org/10.1002/dmrr.2479

[26] Weber, M.A., Mansfield, T.A., Alessi, F., et al. (2016) Effects of Dapagliflozin on Blood Pressure in Hypertensive Diabetic Patients on Renin-Angiotensin System Blockade. Blood Press, 25, 93-103. https://doi.org/10.3109/08037051.2015.1116258

[27] Solini, A., Giannini, L., Vitolo, E., et al. (2017) Dapagliflozin Acutely Improves Endothelial Dysfunction, Reduces Aortic Stiffness and Renal Resistive Index in Type 2 Diabetic Patients: A Pilot Study. Cardiovascular Diabetology, 16, 138. https://doi.org/10.1186/s12933-017-0621-8 
[28] Shigiyama, F., Kumashiro, N., Miyagi, M., et al. (2017) Effectiveness of Dapagliflozin on Vascular Endothelial Function and Glycemic Control in Patients with Early-Stage Type 2 Diabetes Mellitus: Defence Study. Cardiovascular Diabetology, 16, 84. https://doi.org/10.1186/s12933-017-0564-0

[29] Sonesson, C., Johansson, P.A., Johnsson, E., et al. (2016) Cardiovascular Effects of Dapagliflozin in Patients with Type 2 Diabetes and Different Risk Categories: A Meta-Analysis. Cardiovascular Diabetology, 15, 37. https://doi.org/10.1186/s12933-016-0356-y

[30] Petrykiv, S., Sjostrom, C.D., Greasley, P.J., et al. (2017) Differential Effects of Dapagliflozin on Cardiovascular Risk Factors at Varying Degrees of Renal Function. Clinical Journal of the American Society of Nephrology, 12, 751-759. https://doi.org/10.2215/CJN.10180916

[31] Kohan, D.E., Fioretto, P., Johnsson, K., et al. (2016) The Effect of Dapagliflozin on Renal Function in Patients with Type 2 Diabetes. Journal of Nephrology, 29, 391-400. https://doi.org/10.1007/s40620-016-0261-1

[32] Fioretto, P., Stefansson, B.V., Johnsson, E., et al. (2016) Dapagliflozin Reduces Albuminuria over 2 Years in Patients with Type 2 Diabetes Mellitus and Renal Impairment. Diabetologia, 59, 2036-2039. https://doi.org/10.1007/s00125-016-4017-1

[33] Davies, M., Chatterjee, S., Khunti, K., et al. (2016) The Treatment of Type 2 Diabetes in the Presence of Renal Impairment: What We Should Know about Newer Therapies. Clinical Pharmacology, 8, 61-81. https://doi.org/10.2147/CPAA.S82008 